\title{
Enhancement of Link Stability and Connectivity in Vehicular Ad hoc Networks
}

\author{
Kishor N. Tayade ${ }^{1}$, M. U. Kharat ${ }^{2}$ \\ ${ }^{1}$ MET's BKC Institute of Engg., Nashik (Affiliated to Savitribai Phule Pune University, Pune), \\ ${ }^{2}$ MET's BKC Institute of Engineering, Nashik, India, \\ kntayade@rediffmail.com ${ }^{1}$, mukharat@rediffmail.com ${ }^{2}$
}

Article History: Received: 10 November 2020; Revised: 12 January 2021; Accepted: 27 January 2021; Published online: 05 April 2021

\begin{abstract}
Vehicular Ad hoc Networks is a promising sub-group of MANET. VANET is deployed on the highways, where the vehicles are mobile nodes. Safety and intelligent transportation are important VANET applications that require appropriate communication among vehicles, in particular routing technology. VANETs generally inherit their common features from MANETs where vehicles operate in a collaborative and dispersed way for promoting contact among vehicles and with network infrastructure like the Road Side Units (RSU) for enhanced traffic experience. In view of the fast growth of Intelligent Transportation Systems (ITS), VANETs has attracted considerable interest in this decade. VANET suffer from a major problem of link failure due to dynamic mobility of vehicles. In this paper we proposed a position based routing algorithm to identify stable path, this will improve the routing by decreasing overhead and interrupting the number of links. Link Expiration Time (LET) is used to provide the stable link, the link with the longest LET is considered as the most stable link. The multicast Ad-hoc On-demand Distance Vector (MAODV) is proposed to avoid the link breakages by using a link with longest LET. Data loss is reduced by avoiding link breakages and enhance throughput by reducing the communication delay.
\end{abstract}

Keywords: Greedy Forwarding, Link Expiration Time, Mobility Prediction, Routing, VANET

\section{Introduction}

VANET is getting lots of attention from academia and industry because many new applications and services aimed at improving the quality of road traffic and traveler's lives depend on VANET [1]. High mobility and fast topology change characteristics of VANET makes it susceptible to link breakages and data loss due to unstable link connectivity. The problem of communication link instability due to rapid topology changes reduces the reliability of the vehicular communication. It leads to severe security threats [2]. Geo- positioning is one of the applications of intelligent transportation which uses vehicle mobility for broadcasting locations in a explicit city region [3]. VANETs provide different kinds of services by means of communication between roadside units and vehicles as shown in figure 1[4]. In VANETs, a group of several vehicles is built on the basis of similar features. Most commonly VANET uses a Mobility-based clustering approach [5]. High speed of vehicle nodes in VANET leads to poor performance in case of stability and reliability of the cluster [6]. In VANETs, routing is very difficult to form a network topology because of vehicle speed and sometimes leads to network disconnection.

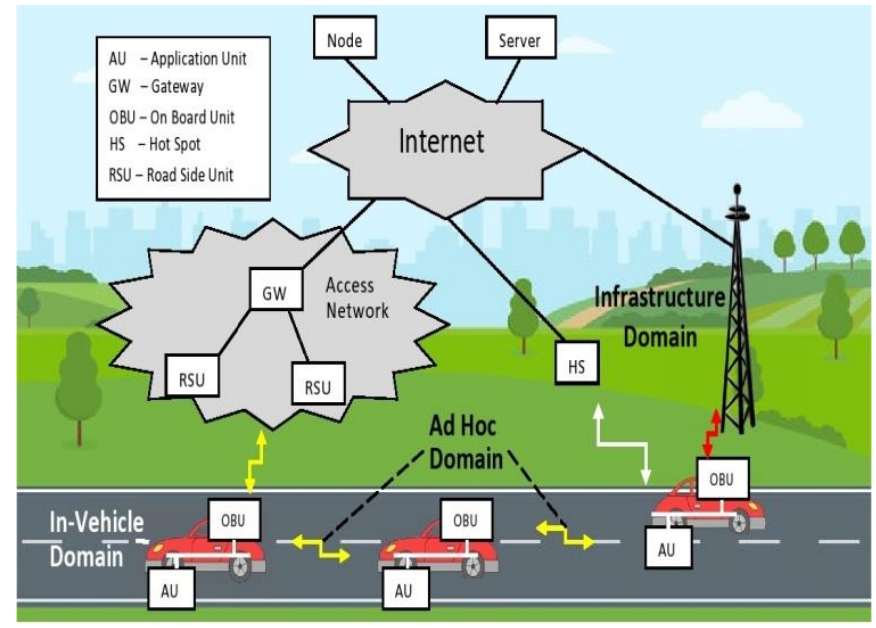

Figure 1. VANET Architecture Diagram

The basic need for the routing protocol is to determine how exactly the router interacts with each other. The information that is being transmitted on the network benefits them by finding routes between two communication link [7]. The routing technique in position based protocol is based upon geographical information of vehicles in motion. Source node uses geographical location of destination node to send a message instead of network 
address [8]. It is best suited routing technique to develop modified routing protocol [9]. The approach preserves network reliability and lowers end-to-end delays and overhead of communications [10]. Communication link life is calculated using Link Expiration Time (LET), it can be also used for marking the link status whether expired or not. Link marked 'expired' will be excluded from routing table to prevent message loss [11]. LET is estimated based upon neighbor's mobility projection [12]. Modified Ad-hoc on demand distance vector protocol is a multicast protocol. Such protocols will improve the performance and maintain minimum transmission latency for real time application [13]. An innovative, greedy-forwarding technique is used for developing modified routing algorithm based upon vehicle location. It reduces connection breakages which leads to stable rout and further enhances throughput and packet delivery ratio [14]. Such implementations need highly efficient connectivity pathways and secure supportive nodes to enable effective data transmission in the estimated timeframe [15]. Architectural diagram of VANET is shown in figure1. Nodes on the road communicate with other nodes by OBU, AU and RSU. As vehicles are moving speedily routing in VANET becomes critical. The vehicular ad hoc network is a self-governing network consisting of an autonomous nodes and the dynamic node mobility results in topology changes. The key goal of the VANET implementation is to enhance road safety by minimizing number of collisions. Nowadays, VANET's deployment has expanded facilities and entertainment to passengers. Mobility prediction of nodes made it easy to approximate stability of the routes in the vehicular ad hoc network. Identifying secure rout helps to enhance routing by minimizing overhead and number of link breakages. Mobility prediction is a significant factor that influences the stability of the connection and the topology of the ad hoc network. As the vehicles are increasing day by day, there is wide scope for the research work on mobility prediction in VANET and it could be valuable in the automobile industry.

\section{Literature Survey}

Number of researchers around the world has done a considerable work on routing and links stability. This survey is based on Vehicular Ad hoc Network (VANET). Mobility prediction algorithm can be used to estimate the path stability in a VANET to prevent accidents. The proposed protocol or approach is used to identify the stable path, provide the stable link and minimize the transmission delay. K. Qureshi et al [16] proposed an enhanced road segment-based geographic routing protocol (ISRP) concentrating upon improved head-node selection for dissemination of information. Algorithms have been developed for full phase of head-node selection and distribution of information between nodes in road-segments. Simulation performed to efficiency of proposed routing system relative to state-of-the-art protocols for dynamic vehicle traffic issues. J. Bang et al [17] suggest a method of accident avoidance supported by the model of node-mobility prediction in VANET using TDMA. Proposed algorithm distributes TDMA time slots to avoid access to reduce collisions by forecasting movement of neighbouring vehicles. It uses control time slot information of occupancy. Results of simulation show the algorithm would minimize collision. M. Ren et al [18] introduces a modern dynamic mobility and stability based clustering technique for city scenario. This technique refers to vehicle's path, relative location and lifetime estimate of the link. Authors contrasted the efficiency of the scheme with Lowest-ID and most-quoted VMaSC clustering algorithm. T. Qiuet al [19] proposes Transmission Mechanism for Emergency Data (TMED) for VANET in that a spider web like broadcast model incorporating electronic maps and GIS is developed. Confirmed-spiders sent to acquire a transmission route from source vehicle to destination vehicle in order to increase the PDR and average latency in emergency data transmission. H. Zhou et al [20] proposed an empirical model to analyze event-driven alert packet transmission mechanism in linked network and thus ensure that it is not easy to ensure that all ZOR nodes are in same partition. A practical model is proposed to analyze eventdriven warning message dissemination mechanism in partitioned networks, further probability of transmitting warning messages to ZOR nodes under various traffic conditions is obtained. I. Abdel-Halim et al [21], suggested a new mobility predictive, effective clustering scheme. The basic principle of MPECS is to split the entire region into different regions using the Voronoi diagram, so that every vehicle would be able to estimate its lifetime, and the expense of cluster head in its existing location. MPECS uses novel combination parameter called the vehicle lifetime value to describe effect of vehicle on cost and cluster stability. Ye, M et al [22] has designed novel mobility prediction based routing protocol (MPBRP) for neighbourhood identification, route recovery and packet transfer in VANET. Many contributions are mentioned in this paper by using driver's intention obtained from GPS. A. Silva et al [23] analyses the vulnerabilities of the GPSR and introduces a new technique called Route Conscious GPSR (PA-GPSR), it contains extra extension tables in neighbor's table to pick right path and avoid the nodes those sent the previous messages in recovery method. In addition, the projected algorithm will reduce packet routing loop by preventing transmission of same packet for same neighbor. These PA-GPSR characteristics can resolve connection break due to inevitable causes, like road accidents. 


\section{Proposed Methodology}

The main goal of the implementation of VANETs is to decrease the number of injuries and enhance road safety. Appropriate vehicle-to-vehicle communication technology, in particular routing technology is required. It transmits the data of one vehicle to another vehicle. Mobility prediction makes it easy to approximate safety of the road traveler by avoiding collisions. A reliable routing protocol would plays significant role to understand dynamic behavior of VANET topology, quality of nodes, and variety of inside factors for the performance of routing protocols. A mobility prediction algorithm is designed to improve the routing, to minimizing the routing failure. In this paper we proposed position based routing protocol to identify stable path, this will improve the routing. The Link Expiration Time (LET) which provides the stable link and the multicast Ad-hoc On-demand Distance Vector (MAODV) is proposed to avoid the link breakages, less data loss and high throughput. A Greedy forwarding approach would be implemented to obtain minimum transmission delay in the packet transfer between vehicles. By using this proposed algorithm and routing protocol, the quality and safety will be obtained with respect to speed, acceleration, traffic related hazards after implementation of vehicle-to-vehicle communication.

Position Based VANET Routing algorithm is proposed to select the stable route, and to minimize the routing failures in the vehicular ad hoc network (VANET). This aims to improve routing by decreasing overhead and interrupting the number of links. Link Expiration Time (LET) is proposed, which is used to provide the stable link, the link with the longest LET is deemed as the most stable link. Multicast Ad-hoc On-demand Distance Vector (MAODV) is introduced to avoid the link breakages. This will reduce the data loss and improve the throughput.

The workflow of the proposed methodology is shown in figure 3. As shown in the diagram source node requests for neighboring nodes for packets. It checks that, if the node is placed on the same road segment, the node communicates a list of points of intersection along the route, so that it can enter the same road segment and direction of source node. If requested node is located on the same road segment then position based routing protocol is used to check if the requested node is from the same group.

If the requested node is from the same group, it indicates high level of stability because both the nodes can be in contact for longer time. If the requested node is not in the same group, then group the nodes with the same direction and find the possible rout. Calculate the link expiration time (LET). If LET is longer, the link is most stable else recompute the LET. The transmission delay required for packet transfer can be calculated. To minimize the transmission delay, Greedy forwarding approach would be used which leads to safe transportation of nodes and improved throughput.

\section{Link Expiration Time}

Here we have considered two vehicles moving with speed $\mathrm{V}_{1}$ and $\mathrm{V}_{2}$, and $\mathrm{R}$ is Line-of-Sight communication range for two vehicles, $\left(\mathrm{X}_{1}, \mathrm{Y}_{1}\right)$ and $\left(\mathrm{X}_{2}, \mathrm{Y}_{2}\right)$, are the positional coordinates and $\theta 1$ and $\theta 2$ are velocity angles as shown in figure 2.

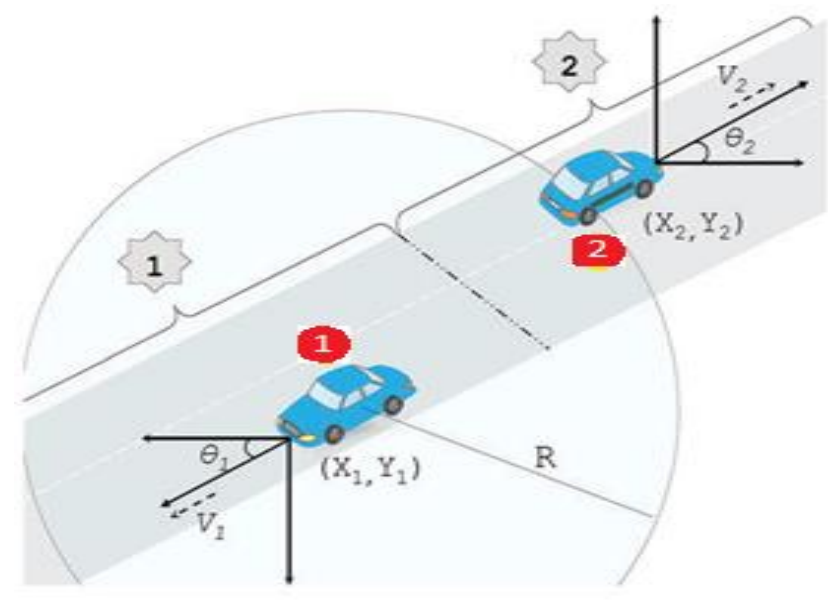

Figure 2. Link Expiration Time Calculation 


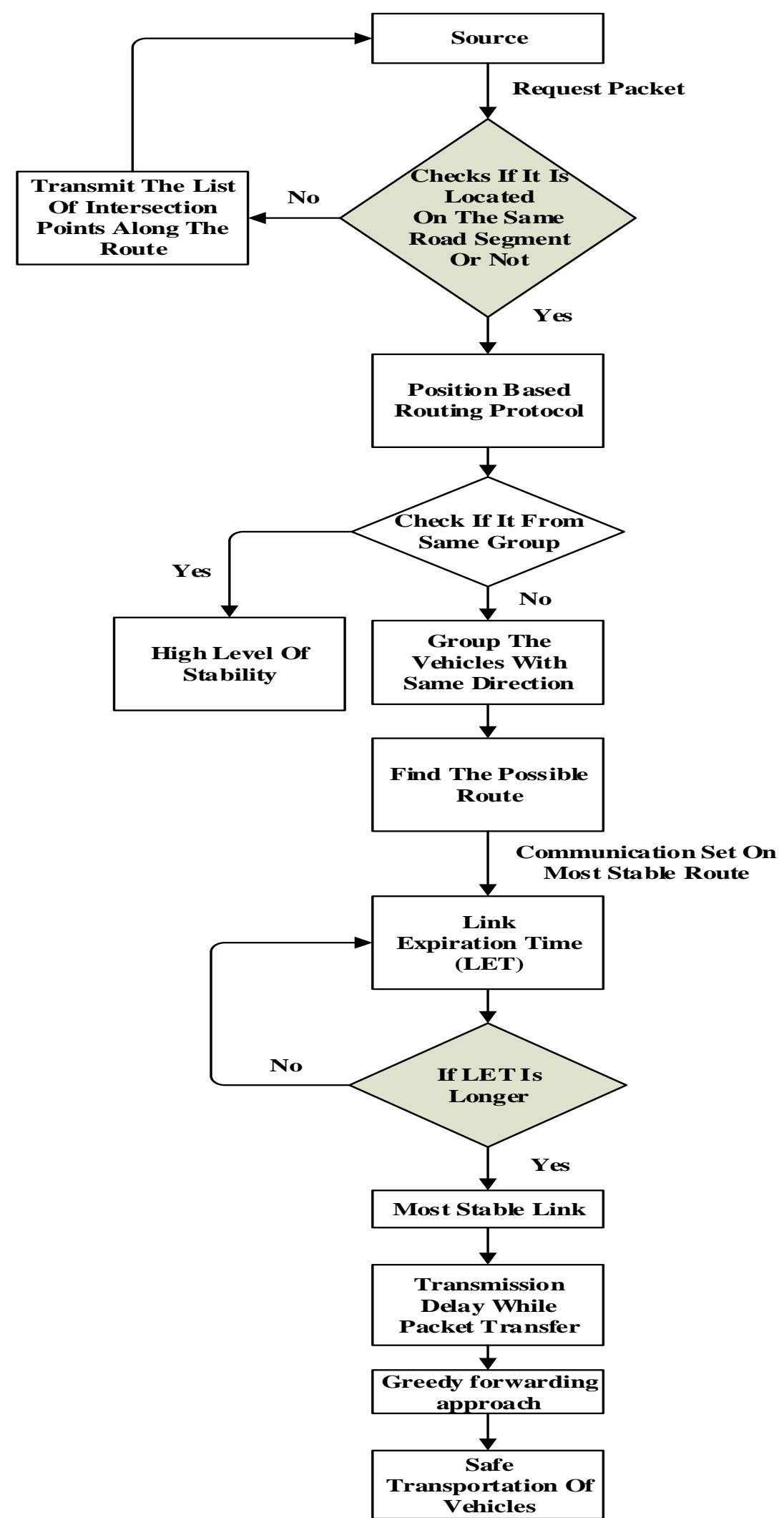

Figure 3. Workflow of Proposed System

Using this information, link estimation of the link between two nodes can be calculated using the equation used in [9] as shown in (1).

$$
\begin{array}{r}
\mathrm{LE}=\frac{-(\mathrm{ps}+\mathrm{q} \mathrm{t})+\sqrt{\left(\mathrm{p}^{2}+\mathrm{q}^{2}\right) \mathrm{R}^{2}-(\mathrm{pt}-\mathrm{sq})^{2}}}{\mathrm{p}^{2}+\mathrm{q}^{2}}, \\
\mathrm{p}=\mathrm{V}_{1} \cos \theta_{1}-\mathrm{V}_{2} \cos \theta_{2},
\end{array}
$$




$$
\begin{aligned}
& \mathrm{q}=\mathrm{V}_{1} \sin \theta_{1}-\mathrm{V}_{2} \sin \theta_{2}, \\
& \mathrm{~s}=\mathrm{X}_{1}-\mathrm{X}_{2}, \\
& \mathrm{t}=\mathrm{Y}_{1}-\mathrm{Y}_{2} .
\end{aligned}
$$

In this methodology, we are using Greedy forwarding method with a view to achieving a minimal transmission delay in the transition of packets between vehicles. This will lead safe transportation of mobile vehicles supported by radio transmission.

\section{Conclusion}

Position based VANET routing algorithm is proposed to enhance the link stability and connectivity by selecting the stable route. The MAODV improves the throughput, and reduce the loss of data. By using proposed method, the transmission delay can be easily reduced by using the stable link and the performance can be enhanced. Packet Delivery Ratio (PDR), throughput can be improved. The simulation of working algorithm would be tested and evaluated by using the NS3, SUMO and python coding.

\section{References}

1. Abdel-Halim and H. Fahmy, "Mobility Prediction in Vehicular Ad-hoc Networks: Prediction Aims, Techniques, Use Cases, and Research Challenges", IEEE Intelligent Transportation Systems Magazine, 2019, pp. 1-1.

2. M. Zhou, L. Han, H. Lu and C. Fu, "Distributed collaborative intrusion detection system for vehicular Ad Hoc networks based on invariant", Computer Networks, vol. 172, p. 107174, 2020.

3. W. Liu and Y. Shoji, "DeepVM: RNN-Based Vehicle Mobility Prediction to Support Intelligent Vehicle Applications", IEEE Transactions on Industrial Informatics, vol. 16, no. 6, 2020pp. 39974006.

4. R. Sultana, J. Grover and M. Tripathi, "Security of SDN-based vehicular ad hoc networks: Stateof-the-art and challenges", Vehicular Communications, 2020 p. 100284.

5. Mukhtaruzzaman and M. Atiquzzaman, "Clustering in vehicular ad hoc network: Algorithms and challenges", Computers \& Electrical Engineering, vol. 88, 2020 p. 106851.

6. D. Zhang, H. Ge, T. Zhang, Y. Cui, X. Liu and G. Mao, "New Multi-Hop Clustering Algorithm for Vehicular Ad Hoc Networks", IEEE Transactions on Intelligent Transportation Systems, vol. 20, no. 4, 2019, pp. 1517-1530.

7. S J. Kamble and M R. Kounte, "Routing and Scheduling Issues in Vehicular Ad-hoc Networks", International Journal of Recent Technology and Engineering, vol. 8, no. 3, 2019.pp. 4272-4283.

8. Nair, C.R., "Analysis and comparative study of topology and position based routing protocols in VANET'. International journal of Engineering Research and general science, vol. 4, no. 1, 2016, pp.43-52.

9. O. Sami Oubbati, N. Chaib, A. Lakas, S. Bitam and P. Lorenz, "U2RV: UAV-assisted reactive routing protocol for VANETs", International Journal of Communication Systems, vol. 33, no. 10, 2019, p. e4104.

10. N. Al-Kharasani, Z. Zukarnain, S. Subramaniam and Z. Hanapi, "An Adaptive Relay Selection Scheme for Enhancing Network Stability in VANETs", IEEE Access, vol. 8, 2020, pp. 128757128765 .

11. O. Rehman, R. Qureshi, M. Ould-Khaoua and M. Niazi, "Analysis of mobility speed impact on end-to-end communication performance in VANETs", Vehicular Communications, vol. 26, 2020, p. 100278.

12. S. Bourebia et al., "A belief function-based forecasting link breakage indicator for VANETs", Wireless Networks, vol. 26, no. 4, 2019pp. 2433-2448.

13. V. Nguyen, T. Khanh, X. Pham, G. Lee and E. Huh, "Performance analysis of adaptive MAC protocol in VANETs considering the potential impact on throughput and transmission delays", International Journal of Communication Systems, vol. 33, 2019, no. 1, p. e4172.

14. A. Bengag, A. Bengag and M. Elboukhari, "A Novel Greedy Forwarding Mechanism Based on Density, Speed and Direction Parameters for Vanets", International Journal of Interactive Mobile Technologies (iJIM), vol. 14, 2020no. 08, p. 196.

15. M. Abhilash, K. Lohitha and K. Patya, "Va-Add - Vehicle and Mobile Assisted Data Delivery in Cluster Based Vehicular Adhoc Networks", Ictact Journal On Communication Technology, Vol. 10, No. 2, 2019, p. 1994-1998. 
16. K. Qureshi, F. Islam, O. Kaiwartya, A. Kumar and J. Lloret, "Improved Road Segment-Based Geographical Routing Protocol for Vehicular Ad-hoc Networks", Electronics, vol. 9, no. 8,2020, p. 1248 .

17. J. Bang and J. Lee, "Collision Avoidance Method Using Vector-Based Mobility Model in TDMABased Vehicular Ad Hoc Networks", Applied Sciences, vol. 10, no. 12, 2020,p. 4181.

18. M. Ren, L. Khoukhi, H. Labiod, J. Zhang and V. Vèque, "A mobility-based scheme for dynamic clustering in vehicular ad-hoc networks (VANETs)", Vehicular Communications, vol. 9, 2017, pp. 233-241.

19. T. Qiu, X. Wang, C. Chen, M. Atiquzzaman and L. Liu, "TMED: A Spider-Web-Like Transmission Mechanism for Emergency Data in Vehicular Ad Hoc Networks", IEEE Transactions on Vehicular Technology, vol. 67, no. 9, 2018, pp. 8682-8694.

20. H. Zhou, S. Xu, D. Ren, C. Huang and H. Zhang, "Analysis of event-driven warning message propagation in Vehicular Ad Hoc Networks", Ad Hoc Networks, vol. 55, 2017,pp. 87-96.

21. I. Abdel-Halim, H. Fahmy and A. Bahaa-El Din, "Mobility prediction-based efficient clustering scheme for connected and automated vehicles in VANETs", Computer Networks, vol. 150, 2019, pp. 217-233.

22. Ye, M., Guan, L. and Quddus, M, "MPBRP-Mobility Prediction Based Routing Protocol in VANETs" In CommNet, April 2019, (pp. 1-7).

23. A. Silva, N. Reza and A. Oliveira, "Improvement and Performance Evaluation of GPSR-Based Routing Techniques for Vehicular Ad Hoc Networks", IEEE Access, vol. 7, 2019,pp. 2172221733.

\section{Authors}

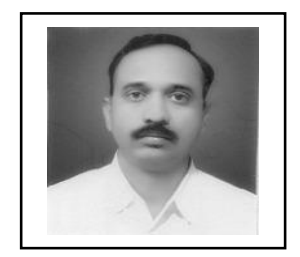

Kishor Tayade has completed his BE and ME from Amravati University, Amravati. Now he is perusing his $\mathrm{PhD}$ from MET's BKC Institute of Engineering, Nashik, Savitribai Phule Pune University, Pune. His area of research interest is vehicular Ad Hoc Networks.

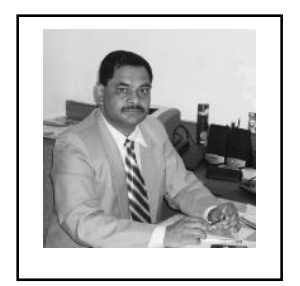

Dr. Madan Kharat is working as Professor and HOD of computer Engineering Department at MET's BKC Institute of Engineering, Nashik. He is having more than 25 years of teaching experience. His area of research interest is Image processing, Natural language processing and Ad Hoc Networks. 